\title{
Oxytocin receptor genetic variation promotes human trust behavior
}

\section{Frank Krueger ${ }^{1,2}{ }^{*}$, Raja Parasuraman ${ }^{2}$, Vijeth lyengar ${ }^{3}$, Matthew Thornburg $^{4}$, Jaap Weel ${ }^{5}$, Mingkuan Lin ${ }^{2}$, Ellen Clarke ${ }^{2}$, Kevin McCabe ${ }^{5}$ and Robert H. Lipsky ${ }^{6}$}

${ }^{1}$ Department of Molecular Neuroscience, George Mason University, Fairfax, VA, USA

${ }^{2}$ Department of Psychology, George Mason University, Fairfax, VA, USA

${ }^{3}$ Department of Psychology and Neuroscience, Duke University, Durham, NC, USA

${ }^{4}$ The College of Humanities and Social Sciences, George Mason University, Fairfax, VA, USA

${ }^{5}$ Department of Economics, George Mason University, Fairfax, VA, USA

${ }^{6}$ Department of Neurosciences, Inova Fairfax Hospital, Inova Health System, Falls Church, VA, USA

\section{Edited by:}

Chad Edward Forbes, University of Delaware, USA

Reviewed by:

Ole A. Andreassen, University of Oslo, Norway

Yang Jiang, University of Kentucky, USA

\section{*Correspondence:}

Frank Krueger, Department of Molecular Neuroscience, Krasnow Institute for Advanced Study, George Mason University, 4400 University Drive, Mail Stop 2A1, Fairfax, VA

22030, USA.

e-mail:fkrueger@gmu.edu
Given that human trust behavior is heritable and intranasal administration of oxytocin enhances trust, the oxytocin receptor (OXTR) gene is an excellent candidate to investigate genetic contributions to individual variations in trust behavior. Although a single-nucleotide polymorphism involving an adenine (A)/guanine (G) transition (rs53576) has been associated with socio-emotional phenotypes, its link to trust behavior is unclear. We combined genotyping of healthy male students $(n=108)$ with the administration of a trust game experiment. Our results show that a common occurring genetic variation (rs53576) in the OXTR gene is reliably associated with trust behavior rather than a general increase in trustworthy or risk behaviors. Individuals homozygous for the G allele (GG) showed higher trust behavior than individuals with A allele carriers (AA/AG). Although the molecular functionality of this polymorphism is still unknown, future research should clarify how the OXTR gene interacts with other genes and the environment in promoting socio-emotional behaviors.

Keywords: trust, oxytocin, social cognition, prosocial behavior, social interaction, oxytocin receptor gene, SNP

\section{INTRODUCTION}

Human societies are probably unique in the extent to which trust characterizes interpersonal interactions. Trust as a critical social process is indispensable in friendship, love, families, and organizations, and it facilitates interpersonal relations and permits reciprocal behaviors that lead to mutual advantages for cooperators during social and economic exchange. In everyday parlance variations in trust are often attributed to attitudes or personality for example, we may describe one person as "very trusting" and another as "so mistrustful." However, trust behavior - the willingness to carry out an action benefiting another person that may leave one vulnerable to some risk of loss - may not be entirely predictable from dispositional factors. Consider a small business owner with an excellent credit history in the current challenging economic environment trying to make an application with a bank for a loan from one of two loan officers. Although the two bank officials may be equally empathetic or altruistic, only one offers to extend a loan. What accounts for the difference in behavior: do genetic factors have a role?

Twin studies have shown that trust behavior is heritable (Cesarini et al., 2008), suggesting that specific genes may be responsible for inter-individual variation. Overlaying the genetics are studies that have sought to understand the role of neuropeptides in the brain such as oxytocin (OXT). Converging animal and human evidence reveals that OXT, a peptide that is produced in the hypothalamus and released in the brain and bloodstream that functions both as a hormone and neurotransmitter, broadly influences socio-emotional behaviors (Lee et al., 2009). Given that intranasal administration of OXT enhances human trust (e.g., Kosfeld et al., 2005; Mikolajczak et al., 2010a,b), there is a clear link between OXT-mediated signaling at the molecular level and this behavior. This biological effect of OXT operates through its cognate receptor, the OXT receptor (OXTR). The OXTR is a 389amino acid polypeptide that modulates a number of behaviors, including stress response, anxiety, social memory and recognition, sexual and aggressive behaviors, and maternal behavior (Lee et al., 2009). Therefore, the OXTR gene is an excellent candidate to investigate genetic contributions to individual variations in trust behavior during social exchange.

The human OXTR gene is located on chromosome 3 p25.3 spanning approximately $19 \mathrm{kbp}$, and is composed of three introns and four exons (Inoue et al., 1994). A single-nucleotide polymorphism (SNP) within intron 3 involving an adenine (A)/guanine (G) transition (rs53576) has recently been associated with different socio-emotional phenotypes (Bakermans-Kranenburg and Van Ijzendoorn, 2008; Tost et al., 2010). For example, A allele carriers (AA and AG) show less dispositional empathy (Rodrigues et al., 2009) compared to those homozygous for the $G$ allele. Despite this accumulating evidence in support of a relationship between OXTR rs53576 and socio-emotional functions, its link to human trust behavior during social interaction remains unknown. To address this open question, we combined a candidate gene approach genotyping for OXTR rs53576 with the administration of a laboratory-based trust game experiment (Berg et al., 
1995). Since the trust game provides the benefits of quantifiability, replicability, and comparability across individuals, it constitutes a more reliable tool for measuring observer-dependent trust behavior than standard self-report questionnaires. We hypothesized that OXTR rs53576 variation relates to individual differences in trust behavior but not in trustworthiness or risk behaviors: Individuals homozygous for the $G$ allele (GG) demonstrate higher levels of trust than A allele carriers (AA/AG). Our hypothesis is based upon the findings that (i) trust behavior is heritable (Cesarini et al., 2008), suggesting that specific genes may be responsible for inter-individual variation; (ii) OXTR rs53576A promotes deficits in socio-emotional phenotypes (Bakermans-Kranenburg and Van Ijzendoorn, 2008; Costa et al., 2009; Lucht et al., 2009; Rodrigues et al., 2009; Tost et al., 2010); and (ii) intranasal administration of OXT enhances human trust behavior rather then trustworthiness or risk behaviors (Kosfeld et al., 2005), for which the biological effect of OXT operates through its OXTR.

\section{MATERIALS AND METHODS SUBJECTS}

In this study, 108 healthy right-handed male students (European ancestry, "European Americans," mean \pm SD, age: $20.2 \pm 2.2$ years, education: $14.8 \pm 1.9$ years) gave written consent before participating for financial compensation in the neurobehavioral protocol approved by the George Mason University Human Subjects Research Board.

\section{PROCEDURE}

The neurobehavioral protocol included a laboratory-based experiment (Berg et al., 1995) measuring trust, trustworthiness, and risk behavior as well as psychological control measures accounting for a possible confound of the OXTR rs53576 gene polymorphism on trust behavior.

The laboratory-based experiment included a two-person investment game measuring trust and trustworthiness behavior and a lottery game measuring risk behavior. In the investment game, two interacting participants receive an initial endowment of 10 monetary units (MU) and one participant (the investor) decides how much money to send to another participant (the trustee). The sent amount (a measure of trust: $0,1,2, \ldots, 10 \mathrm{MU}$ ) is then tripled, and the trustee decides how much of the money received to send back to the investor. The investor's final payoff equals the initial endowment minus the transfer to the trustee, plus the back transfer from the trustee. The trustee's final payoff equals the initial endowment plus the tripled transfer of the investor, minus the back transfer to the investor. In a group session of 12 subjects, participants made five decisions each in the roles of investors and trustees while paired with different, randomly selected interaction partners, and received feedback about their partners' decisions.

In the lottery game, participants made decisions only as investors who could transfer up to $10 \mathrm{MU}$ into a lottery rather than to a trustee embedded in a social interaction. A computer chose investment return equal to the payoff structure in the trust game at any feasible back transfer level. Investors in the trust and risk games faced the same objective risks, ensuring that trusting behavior could be distinguished from risk behavior. The earned
MUs were exchanged into real money according to a publicly announced exchange rate ( $40 \mathrm{MUs}=\$ 1$ ).

After the laboratory-based experiment, participants completed psychological control measures to account for possible confounds of the OXTR rs53576 gene polymorphism on trust behavior. Control measures included empathy (interpersonal reactivity index, IRI, five-point Likert scale; Davis, 1983), altruism (Rushton altruism scale, RAS, five-point Likert Scale; Rushton et al., 1981), theory of mind ability (reading the mind in the eyes test, RMET, multiplechoice test; Baron-Cohen et al., 2001), attachment style (Relationship Scale Questionnaire, RSQ, five-point Likert scale; Griffin and Bartholomew, 1994), and personality style (NEO five-factor inventory, NEO-FFI, five-point Likert scale; Costa and McCrae, 1992; see Appendix).

\section{GENOTYPING ANALYSIS}

The SNP that was the focus of this study (rs53576) is located within a region of the OXTR gene having extensive linkage disequilibrium (LD; Figure 1). Participants provided saliva buccal swabs for genotyping, which were collected in lysis buffer $(100 \mathrm{mM} \mathrm{NaCl}$, $10 \mathrm{mM}$ EDTA, $10 \mathrm{mM}$ Tris $\mathrm{pH} 8,0.1 \mathrm{mg} / \mathrm{ml}$ proteinase $\mathrm{K}$, and $0.5 \% \mathrm{w} / \mathrm{v}$ SDS $)$ at $-20^{\circ} \mathrm{C}$ until further processing using an Isohelix DNA isolation kit (Harrietsham, Kent, UK).Genotyping was performed by a PCR-coupled DNA melting analysis method (Lipsky et al., 2001) using SsoFast EvaGreen PCR mix (Bio-Rad Laboratories, Hercules, CA, USA). Amplification primer sequences were: forward 5'-GAATAGGGACTTTCTAAGCA-3'; reverse 5' GTCCATCTAATTGTGATTTGT-3'. Approximately $5 \mathrm{ng}$ DNA from each sample were plated in 96-well plates for genotyping with $10 \%$ randomly duplicated samples for detecting genotyping error. Genotyping was performed using a Bio-Rad CFX96 Sequence Detector with a 96-well plate format. The overall genotyping accuracy was $100 \%$ with $100 \%$ completion. The genotype distribution $(\mathrm{GG}=56, \mathrm{AG}=43, \mathrm{AA}=9)$ met Hardy-Weinberg Equilibrium expectations $\left[\chi^{2}(1)=0.03, P=0.854\right]$ and groups were matched on age (GG: $20.3 \pm 2.5$, AG: $19.9 \pm 1.8$, AA: $20.4 \pm 1.1$; $F(2,107)=0.63, P=0.533)$ and education $[\mathrm{GG}: 15.1 \pm 2.1, \mathrm{AG}$ : $14.6 \pm 1.8$, AA: $14.8 \pm 1.3 ; F(2,107)=0.81, P=0.446]$. We used a dominant model to increase the statistical power in our study by collapsing the AA and AG groups, because prior evidence suggests that rs53576A promotes deficits in socioemotional behavior and AA homozygotes were rare in our study as previously reported (Bakermans-Kranenburg and Van Ijzendoorn, 2008; Costa et al., 2009; Rodrigues et al., 2009). A prospective power calculation showed that with the current sample size there was greater than $80 \%$ power to detect an association with moderate to large effects sizes (Cohen's $d>0.5)$.

\section{DATA ANALYSIS}

Data analysis was carried out using SPSS 14.0 (SPSS Inc., Chicago, USA) with alpha set to $P<0.05$ (two-tailed). First, the genotype effects on relative frequency of investor's transfers in the trust experiment and risk experiments were determined using a mixed $11 \times 2$ analysis of variance (ANOVA) with transfer level $(0,1,2$, $\ldots, 10 \mathrm{MU}$ ) as a within-subjects factor and group (GG, AG/AA) as a between-subjects factor. Second, the genotype effect on relative 
frequency of trustee's back transfers in the trust experiment were determined using a mixed $11 \times 30 \times 2$ ANOVA with transfer level $(0,1,2, \ldots, 10 \mathrm{MU})$ and back transfer level $(0,1,2, \ldots, 30 \mathrm{MU})$ as a within-subjects factors and group (GG, AG/AA) as a betweensubjects factor. Third, planned follow-up Pearson Chi-square tests (applying Bonferroni correction) were performed to compare relative frequencies of money transfer between groups for each transfer and back transfer level. In addition, effect sizes (Cohen's $d$ ) were calculated representing the observed genotype group effects ( $d=0.2$ indicates a small effect size, $d=0.5$ a medium effect size, and $d=0.8$ large effect size; Cohen, 1988). Finally, genotype group effects on psychological control measures were determined using independent-samples $t$-tests to rule out alternative explanations due to empathy, altruism, theory of mind ability, attachment style, and personality style.

\section{RESULTS}

The rs53576 SNP of the OXTR gene was specifically associated with trusting behavior rather than a general increase in risk or trustworthy behaviors. For the investor's transfers in the trust experiment, the ANOVA revealed no significant main effect for group $[F(1,8)=0.24, P=0.636]$, but a significant main effect for transfer level $[F(10,80)=216.45, P<0001]$, and a significant interaction effect for transfer level $\times$ group $[F(10,80)=6.02, P<0.001]$. The interaction effect was driven by an only significantly higher money transfer for the maximal trust level: the GG group transferred 14\% more money than the AG/AA group $\left[\chi^{2}=11.9, P<0.0001, P\right.$ corrected for multiple testing $<0.001$, Cohen's $d=0.7$; Note that the AG and AA groups did not differ in their money transfers at the maximal trust level (AG: $0.41 \pm 0.04$, AA: $0.44 \pm 0.05, \chi^{2}=0.2, P=0.706$; Figure 2A)].

For the investor's transfers in the risk experiment, the ANOVA revealed only a significant main effect for transfer level $[F(10,180)=333.24, P<0.001]$, but no significant main effect for group $[F(1,18)=1.65, P=0.215]$, and no significant interaction effect for transfer level $\times$ group $[F(10,180)=1.83, P=0.119$; Figure 2B].

\section{Chromosome 3p25.3}

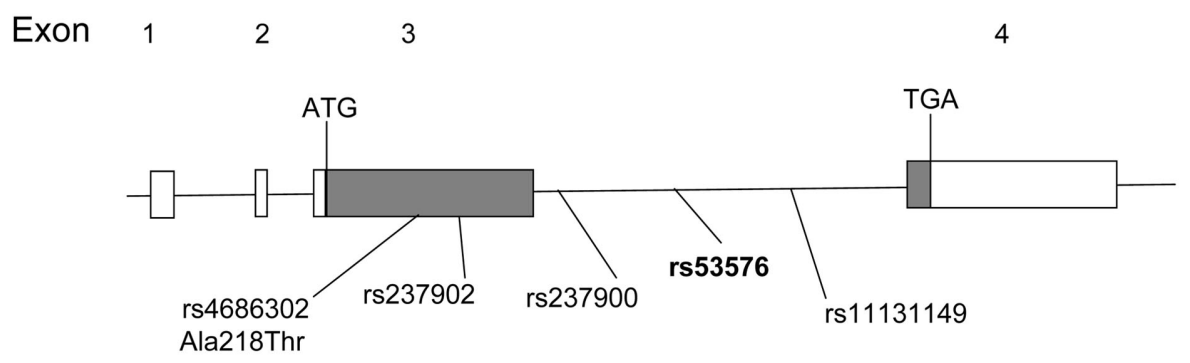

$$
\text { LD (D'-values = 1) }
$$

Size

$$
\sim 10 \mathrm{kbp}
$$

FIGURE 1 | Schematic of the human OXTR gene. Showing location of the four exons, the five SNP marker positions (including rs53576), and known LD block (from HapMap data release February 27, 2009, NCBI B36 assembly).

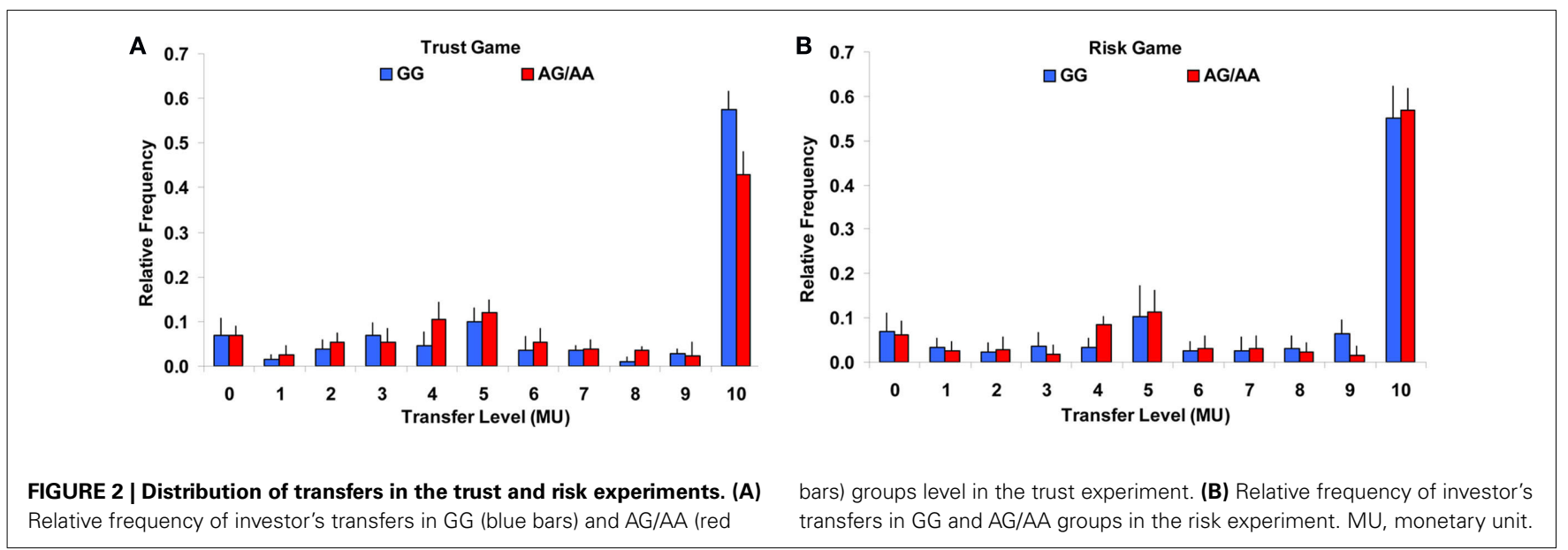


For the trustee's back transfers in the trust experiment, the ANOVA revealed only significant main effects for transfer level $[F(10,180)=65.03, P<0.001]$ as well as back transfer level $[F(30,540)=20.04, P<0.001]$ and a significant interaction effect for transfer level $\times$ back transfer level $[F(300,5400)=20.84, P<0.001]$, but no significant main effect for group $[F(1,18)=0.48, P=0.497]$, and no significant interaction effects for transfer level $\times$ group $[F(10,180)=1.30, P=$ $0.231]$, back transfer level $\times$ group $[F(30,540)=1.28, P=0.217]$, and transfer level $\times$ back transfer level $\times$ group $[F(30,540)=1.24$, $P=0.269$; Figure 3; see Table A1 in Appendix for trustee's back transfer for each level; Figure A1 for distribution.].

Differences on psychological control measures (mean \pm SD) were ruled out as alternative explanations, including altruism (RAS: $55.8 \pm 1.4,58.2 \pm 1.9 ; P=0.232$ ), theory of mind ability (RMET: $72.5 \pm 1.3,72.8 \pm 1.5 \%, P=0.847$ ), attachment styles (RSQ, secure: $3.3 \pm 0.1,3.2 \pm 0.1$; fearful: $2.7 \pm 0.1,2.9 \pm 0.1$; preoccupied: $2.9 \pm 0.1,2.8 \pm 0.1$; dismissing: $3.1 \pm 0.1,3.1 \pm 0.1$; Ps $>0.172$ ), and personality styles (NEO-FFI, neuroticism: $53.3 \pm 0.8,55.6 \pm 0.9$; extraversion: $52.8 \pm 0.9,53.2 \pm 1.0$; openness: $45.8 \pm 0.8,46.0 \pm 0.8$; agreeableness: $42.6 \pm 1.1,45.5 \pm 1.4$; conscientiousness: $41.1 \pm 0.8,40.9 \pm 0.9$; Ps $>0.120)$. Although the GG group had higher dispositional empathy than the AG/AA group [IRI: $2.7 \pm 0.1,2.5 \pm 0.1 ; t(106)=1.8, P=0.039$, one-sided, Cohen's $d=2.0$ ], empathy was not associated with trust, risk, and trustworthy behaviors $(r s<0.11, P s>0.512)$.

\section{DISCUSSION}

The goal of the study was to investigate the relationship between OXTR rs53576 and human trust by combining a candidate gene approach genotyping for OXTR rs53576 and the administration of a laboratory-based trust game experiment. We demonstrated that a common occurring genetic variation (rs53576) in the OXTR gene is reliably associated with trust behavior rather than a general increase in trustworthy or risk behaviors.

Our results extends previous knowledge showing that OXTR rs53576A promotes deficits in self-reported socio-emotional measures such as empathy (Rodrigues et al., 2009), attachment (Costa et al., 2009), positive affect (Lucht et al., 2009), maternal sensitivity
(Bakermans-Kranenburg and Van Ijzendoorn, 2008), and prosocial temperament (Tost et al., 2010). In our study, participants with A allele carriers (AA/AG) showed lower trust behavior than participants homozygous for the G allele (GG). Notably, the AG/AA group had lower dispositional empathy than the GG group, replicating previous research: mothers with the AA or AG genotype of rs53576 showed lower levels of sensitive responsiveness (presupposing awareness of and empathy with children's needs) toward their toddlers (Bakermans-Kranenburg and Van Ijzendoorn, 2008) and individuals with one or two copies of the A allele exhibited both lower behavioral and dispositional empathy (Rodrigues et al., 2009). Importantly, in our study dispositional empathy was not associated with trust, risk, and trustworthy behaviors. In addition, alternative explanations could be ruled out such as altruism, theory of mind ability, attachment styles (secure, fearful, preoccupied, and dismissing), and personality styles (neuroticism, extraversion, openness, and agreeableness).

Our results are further complemented by evidence that OXTR rs53576 impacts both hypothalamic-limbic structure and function (Tost et al., 2010). A recent neuroimaging study has shown a volume reduction in the hypothalamus in carriers of the OXTR rs53576A (Tost et al., 2010), which is associated with an increased risk for autistic spectrum disorders characterized by qualitative abnormalities in reciprocal social interaction and communication (Wu et al., 2005; Liu et al., 2010; Wermter et al., 2010). The hypothalamus is the primarily region for the synthesis of OXT which is then released into the brain and bloodstream to function both as a hormone and neurotransmitter (Lee et al., 2009). Converging evidence from animal studies has shown that the OXT system broadly influences social behavior such as pair bonding/attachment, peer recognition, and social memory (Lee et al., 2009; Ebstein et al., 2010). For example, OXT receptor knockout mice demonstrate several aberrations in social behavior, including aggression and mother-offspring interaction (Nishimori et al., 2008) that can be fully restored by injection of OXT (Ferguson et al., 2001). Since neuropeptides cross the blood-brain barrier after intranasal administration (Born et al., 2002), OXT can also be used in humans to investigate its effects on the central nervous system (Heinrichs and Domes, 2008). Recent studies have demonstrated that OXT is a
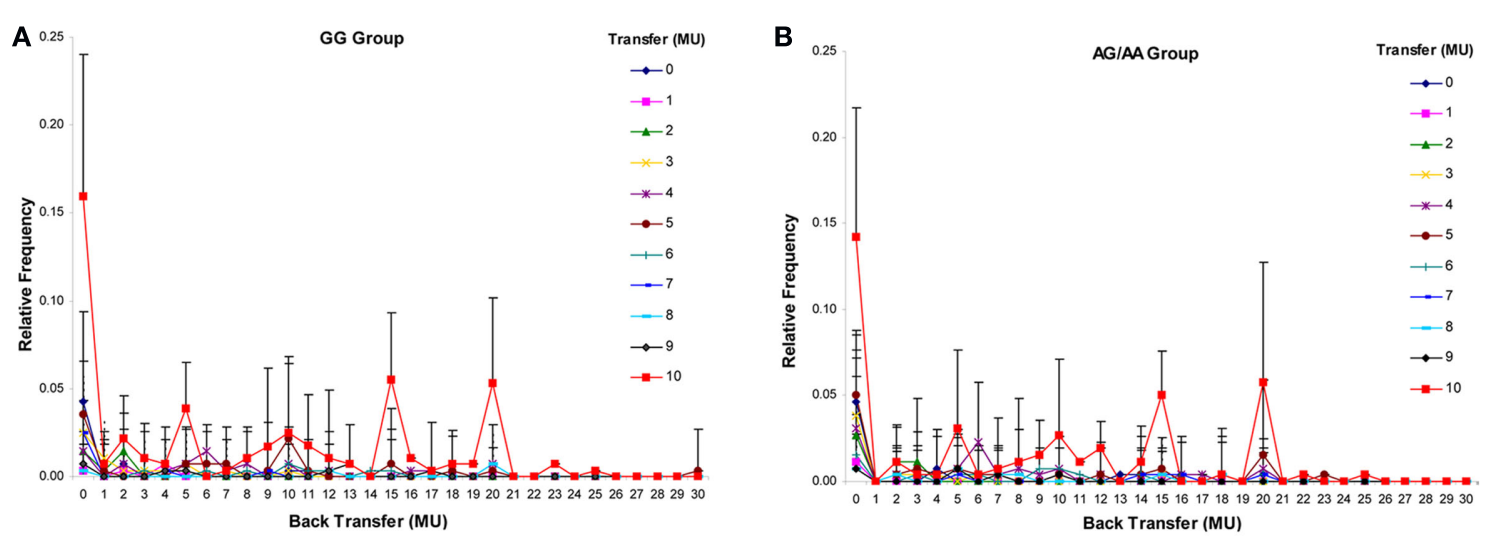

FIGURE 3 | Distribution of back transfers in the trust experiment. (A) Relative frequency of trustees' back transfers in the GG group. (B) Relative frequency of trustee's back transfers in the AG/AA group. MU, monetary unit. 
crucial mediator in the regulation of complex social cognition and behavior (for reviews, see Bartz et al., 2011; Kemp and Guastella, 2011; Meyer-Lindenberg et al., 2011). In particular, administration of exogenous OXT increases trust behavior (e.g., Kosfeld et al., 2005; Mikolajczak et al., 2010a,b) as well as shapes the neural circuitry of trust and trust adaptation (Baumgartner et al., 2008), recently confirmed by a meta-analysis on exogenous OXT trust studies (Van Ijzendoorn and Bakermans-Kranenburg, 2011).

Our results contrast with the recent negative finding between rs53576 and human trust (Apicella et al., 2010). We argue that this discrepancy in results can be explained by differences in important methodological features between the two experiments. Apicella et al. (2010) administered the standard trust game (Berg et al., 1995), in which participants first played the role of trustor and then trustee albeit with a different anonymous partner. However, to elicit the trustworthiness of the trustee, they used a strategy method in which participants were asked to indicate how they would react to any possible amount sent prior to observing trustor behavior (Camerer, 2003). Previous research has shown that when using this method participants play differently when making economic decisions, i.e., they are playing the population but not the person and this may have a profound effect on their prosocial behavior (Rapoport, 1997; McCabe et al., 2000). Importantly, a recent meta-analysis reviewing trust behavior (i.e., 84 replications of the standard trust game across 29 countries with widely varying cultures with an average of 140 players in each of these replications for a total of 11,913 participants) revealed that approximately $40 \%$ of the variance in trust behavior is due to changes in the experimental protocols by manipulating methodological key variables influencing human trust (Johnson and Mislin, 2009).

Further, Apicella et al. (2010) tested both male and female participants for which the vast majority of their participants were females (approximately $80 \%$, see for detailed description Cesarini et al., 2009). We only investigated male-male pairs in our study because of the evidence that female-female pairs exhibit lower trust than male pairs (Croson and Buchan, 1999). Interestingly, by breaking down their results into sex specific effects for OXT rs53576, the authors demonstrated statistical significance at the $10 \%$ level for the male but not for the female participants. Moreover, Apicella et al. (2010) only considered as their measure of trust behavior the overall amount of money transferred but not the money transferred for each trust level separately. However, a recent exogenous OXT study by Kosfeld et al. (2005) demonstrated that the enhancement of trust is driven by a higher frequency of money transfers for the maximal trust level. The same effect was revealed in our study but was not considered as an outcome measure in Apicella et al. (2010). Overall, these crucial factors might explain why Apicella et al. (2010) failed to detect an association between rs53576 and human trust. Because of discrepant findings and the risk of our current findings being false positive, future studies have to clarify the specific effects of those factors on the relationship between OXTR rs53576 and human trust in the context of the standard trust game.

There are limitations in our study that deserve discussion. First, our sample size was modest so that our findings could be due to the "winners curse" effect based on the fact that original studies tend to overestimate effect sizes (Zöllner and Pritchard,
2007). However, our study was a theoretically driven, candidate gene investigation with a reduction of population stratification by testing only a Caucasian sample. Instead of conducting a "fishing expedition" in which a problem with spurious associations could arise (as in multiple SNP or GWAS studies), we hypothesized an association with one specific phenotype but not others based on previous published association studies (e.g., Rodrigues et al., 2009; Tost et al., 2010). Importantly, our results fit with the literature on the directionality of the association between OXTR rs53576 and other socio-emotional behaviors providing evidence that OXTR rs53576A promotes deficits in socio-emotional phenotypes (Bakermans-Kranenburg and Van Ijzendoorn, 2008; Costa et al., 2009; Lucht et al., 2009; Rodrigues et al., 2009; Tost et al., 2010). In only one association study, the AA genotype in children with ADHD was associated with better social ability compared to the AG genotype but not with the GG genotype (Park et al., 2010).

Second, we collapsed in our study the AA and AG groups using a dominant model to improve statistical power, since prior evidence suggests that rs53576A promotes deficits in socio-emotional behavior and AA homozygotes were rare in our population. The same approach was done in previous studies demonstrating that individuals with the GG genotype, relative to those with the A allele, exhibit more sensitive parenting behavior (BakermansKranenburg and Van Ijzendoorn, 2008), empathy (Rodrigues et al., 2009), and attachment (Costa et al., 2009). In contrast, other studies combined the GG/AG groups and demonstrated that individuals with the GG/AG genotypes (compared with those with the AA genotype) seek more emotional social support (Kim et al., 2010) and show higher positive affect (Lucht et al., 2009). Finally, another study revealed that allelic variation in OXTR rs53576 predicts differences in prosocial temperament by showing a difference only between the two homozygote genotype groups (G/G vs. A/A): the GG group showed higher scores in prosocial temperament compared to the AG group (Tost et al., 2010). Because of different group combinations, future association studies should attempt to replicate our findings across populations using larger independent-samples so that all three genotypes (AA/AG/GG) can be compared directly.

In conclusion, the present study demonstrates that $\mathrm{G}>\mathrm{A}$ SNP, rs53576, in the OXTR gene is associated with observer-dependent trust behavior during social interaction. Because the molecular functionality of OXTR rs53576 is still unknown, our results cannot exclude the possibility that the observed effect may reflect the impact of OXTR variants in LD with rs53576. However, the position of this polymorphism within intron 3 suggests involvement in direct gene-gene communication (Mattick and Gagen, 2001), and presents an avenue for future research determining how the OXTR gene interacts with other genes and the environment in promoting socio-emotional behaviors in both nonclinical and clinical populations. Although our study presents the first evidence indicating a role of OXTR rs53576 in human trust behavior, however, cautious replication is needed given the problem of replication validity and risk of false positive results in genetic association studies (Ioannidis et al., 2001). Future studies should replicate those finding in larger independentsamples with similar experimental designs and apply quantitative 
approaches such as meta-analyses to combine the results of various studies on the same phenotype to explain and estimate their diversity.

\section{AUTHORS CONTRIBUTIONS}

Frank Krueger, Raja Parasuraman, Kevin McCabe, and Robert H. Lipsky designed research; Frank Krueger, Vijeth Iyengar, Matthew Thornburg, and Jaap Weel performed research; Frank Krueger, Mingkuan Lin, Ellen Clarke, and Robert H. Lipsky analyzed data;

\section{REFERENCES}

Apicella, C. L., Cesarini, D., Johannesson, M., Dawes, C. T., Lichtenstein, P., Wallace, B., Beauchamp, J., and Westberg, L. (2010). No association between oxytocin receptor (OXTR) gene polymorphisms and experimentally elicited social preferences. PLOS ONE 5, el1153. doi:10.1371/journal.pone.0011153

Bakermans-Kranenburg, M. J., and Van Ijzendoorn, M. H. (2008). Oxytocin receptor (OXTR) and serotonin transporter (5-HTT) genes associated with observed parenting. Soc. Cogn. Affect. Neurosci. 3, 128-134.

Baron-Cohen, S., Wheelwright, S., Hill, J., Raste, Y., and Plumb, I. (2001). The "reading the mind in the eyes" test revised version: a study with normal adults, and adults with Asperger syndrome or high-functioning autism. J. Child. Psychol. Psychiatry 42, 241-251.

Bartz, J. A., Zaki, J., Bolger, N., and Ochsner, K. N. (2011). Social effects of oxytocin in humans: context and person matter. Trends Cogn. Sci. (Regul. Ed.) 15, 301-309.

Baumgartner, T., Heinrichs, M., Vonlanthen, A., Fischbacher, U., and Fehr, E. (2008). Oxytocin shapes the neural circuitry of trust and trust adaptation in humans. Neuron 58, 639-650.

Berg, J., Dickhaut, J., and Mccabe, K. (1995). Trust, reciprocity, and social history. Games Econ. Behav. 10, 122-142.

Born, J., Lange, T., Kern, W., Mcgregor, G. P., Bickel, U., and Fehm, H. L. (2002). Sniffing neuropeptides: a transnasal approach to the human brain. Nat. Neurosci. 5, 514-516.

Camerer, C. F. (2003). Behavioral Game Theory. Princeton: Princeton University Press.

Cesarini, D., Dawes, C. T., Fowler, J. H., Johannesson, M., Lichtenstein, P., and Wallace, B. (2008). Heritability of cooperative behavior in the trust game. Proc. Natl. Acad. Sci. U.S.A. 105, 3721-3726.

Cesarini, D., Dawes, C. T., Johannesson, M., Lichtenstein, P., and Wallace, B.
(2009). Genetic variation in preferences for giving and risk taking. Q. J. Econ. 124, 809-842.

Cohen, J. (1988). Statistical Power Analysis for the Behavioral Sciences. Hillsdale, NJ: Lawrence Erlbaum Associates.

Costa, B., Pini, S., Gabelloni, P., Abelli, M., Lari, L., Cardini, A., Muti, M., Gesi, C., Landi, S., Galderisi, S., Mucci, A., Lucacchini, A., Cassano, G. B., and Martini, C. (2009). Oxytocin receptor polymorphisms and adult attachment style in patients with depression. Psychoneuroendocrinology 34, 1506-1514.

Costa, P. T., and McCrae, R. R. (1992). Revised NEO Personality Inventory (NEO PI-R) Professional Manual. Odessa, FL: Psychological Assessment Resources, Inc.

Croson, R., and Buchan, N. (1999). Gender and culture: international experimental evidence from trust games. Am. Econ. Rev. 89, 386-391.

Davis, M. (1983). Measuring individual differences in empathy: evidence for a multidimensional approach. J. Pers. Soc. Psychol. 44, 113-126.

Ebstein, R. P., Israel, S., Chew, S. H., Zhong, S., and Knafo, A. (2010). Genetics of human social behavior. Neuron 65, 831-844.

Ferguson, J. N., Aldag, J. M., Insel, T. R., and Young, L. J. (2001). Oxytocin in the medial amygdala is essential for social recognition in the mouse. $J$. Neurosci. 21, 8278-8285.

Griffin, D., and Bartholomew, K. (1994). Models of the self and other: fundamental dimensions underlying measures of adult attachment. J. Pers. Soc. Psychol. 67, 430-445.

Heinrichs, M., and Domes, G. (2008). Neuropeptides and social behaviour: effects of oxytocin and vasopressin in humans. Prog. Brain Res. 170, 337-350.

Inoue, T., Kimura, T., Azuma, C., Inazawa, J., Takemura, M., Kikuchi, T., Kubota, Y., Ogita, K., and Saji, F. (1994). Structural organization of the human oxytocin receptor gene. J. Biol. Chem. 269, 32451-32456.

and Frank Krueger, Raja Parasuraman, and Robert H. Lipsky wrote the paper.

\section{ACKNOWLEDGMENTS}

We gratefully acknowledge all of the students who participated in this project. This research was supported by the MasonInova Fund (to Frank Krueger, Kevin McCabe, and Robert H. Lipsky) and by Air Force Office of Sponsored Research grant FA9550-10-1-0385 to R. Parasuraman.

Ioannidis, J. P., Ntzani, E. E., Trikalinos, T. A., and Contopoulos-Ioannidis, D. G. (2001). Replication validity of genetic association studies. Nat. Genet. 29, 306-309.

Johnson, N. D., and Mislin, A. (2009). Cultures of kindness: a meta-analysis of trust game experiments. Available at: http://ssrn.com/abstract $=1315325$

Kemp, A., and Guastella, A. (2011). The role of oxytocin in human affect: a novel hypothesis. Psychol. Sci. 20, 222-231.

Kim, H. S., Sherman, D. K., Sasaki, J. Y., Xu, J., Chu, T. Q., Ryu, C., Suh, E. M., Graham, K., and Taylor, S. E. (2010). Culture, distress, and oxytocin receptor polymorphism (OXTR) interact to influence emotional support seeking. Proc. Natl. Acad. Sci. U.S.A. 107, 15717-15721.

Kosfeld, M., Heinrichs, M., Zak, P. J., Fischbacher, U., and Fehr, E. (2005). Oxytocin increases trust in humans. Nature 435, 673-676.

Lee, H. J., Macbeth, A. H., Pagani, J. H., and Young, W. S. III. (2009). Oxytocin: the great facilitator of life. Prog. Neurobiol. 88, 127-151.

Lipsky, R. H., Mazzanti, C. M., Rudolph, J. G., Xu, K., Vyas, G., Bozak, D., Radel, M. Q., and Goldman, D. (2001). DNA melting analysis for detection of single nucleotide polymorphisms. Clin. Chem. 47, 635-644.

Liu, X., Kawamura, Y., Shimada, T., Otowa, T., Koishi, S., Sugiyama, T., Nishida, H., Hashimoto, O., Nakagami, R., Tochigi, M., Umekage, T., Kano, Y., Miyagawa, T., Kato, N., Tokunaga, K., and Sasaki, T. (2010). Association of the oxytocin receptor (OXTR) gene polymorphisms with autism spectrum disorder (ASD) in the Japanese population. J. Hum. Genet. 55, 137-141.

Lucht, M. J., Barnow, S., Sonnenfeld, C., Rosenberger, A., Grabe, H. J., Schroeder, W., Volzke, H., Freyberger, H. J., Herrmann, F. H., Kroemer, H., and Rosskopf, D. (2009). Associations between the oxytocin receptor gene (OXTR) and affect, loneliness and intelligence in normal subjects. Prog. Neuropsychopharmacol. Biol. Psychiatry 33, 860-866.

Mattick, J. S., and Gagen, M. J. (2001). The evolution of controlled multitasked gene networks: the role of introns and other noncoding RNAs in the development of complex organisms. Mol. Biol. Evol. 18, 1611-1630.

McCabe, K., Smith, V., and Lepore, M. U. (2000). Intentionality detection and "mindreading": why does game form matter? Proc. Natl. Acad. Sci. U.S.A 4404-4409.

Meyer-Lindenberg, A., Domes, G., Kirsch, P., and Heinrichs, M. (2011) Oxytocin and vasopressin in the human brain: social neuropeptides for translational medicine. Nat. Rev. Neurosci. 12, 524-538.

Mikolajczak, M., Gross, J. J., Lane, A., Corneille, O., De Timary, P., and Luminet, O. (2010a). Oxytocin makes people trusting, not gullible. Psychol. Sci. 21, 1072-1074.

Mikolajczak, M., Pinon, N., Lane, A., De Timary, P., and Luminet, $O$. (2010b). Oxytocin not only increases trust when money is at stake, but also when confidential information is in the balance. Biol. Psychol. 85, 182-184.

Nishimori, K., Takayanagi, Y., Yoshida, M., Kasahara, Y., Young, L. J., and Kawamata, M. (2008). New aspects of oxytocin receptor function revealed by knockout mice: sociosexual behaviour and control of energy balance. Prog. Brain Res. 170, 79-90.

Park, J., Willmott, M., Vetuz, G., Toye, C., Kirley, A., Hawi, Z., Brookes, K. J., Gill, M., and Kent, L. (2010). Evidence that genetic variation in the oxytocin receptor (OXTR) gene influences social cognition in ADHD. Prog. Neuropsychopharmacol. Biol. Psychiatry 34, 697-702.

Rapoport, A. (1997). Order of play in strategically equivalent games in extensive form. Int. J. Game Theory 26, 113-136. 
Rodrigues, S. M., Saslow, L. R., Garcia, N., John, O. P., and Keltner, D. (2009). Oxytocin receptor genetic variation relates to empathy and stress reactivity in humans. Proc. Natl. Acad. Sci. U.S.A. 106, 21437-21441.

Rushton, J., Chrisjohn, R., and Fekken, G. (1981). The altruistic personality and the self-report altruism scale. Pers. Individ. Dif. 50, 1192-1198.

Tost, H., Kolachana, B., Hakimi, S., Lemaitre, H., Verchinski, B. A., Mattay, V. S., Weinberger, D. R., and Meyer-Lindenberg, A. (2010). A common allele in the oxytocin receptor gene (OXTR) impacts prosocial temperament and human hypothalamic-limbic structure and function. Proc. Natl. Acad. Sci. U.S.A. 107, 13936-13941.

Van Ijzendoorn, M. H., and BakermansKranenburg, M. J. (2011). A sniff of trust: meta-analysis of the effects of intranasal oxytocin administration on face recognition, trust to in-group, and trust to out-group. Psychoneuroendocrinology. PMID: 21802859. [Epub ahead of print].

Wermter, A. K., Kamp-Becker, I., Hesse, P., Schulte-Korne, G., Strauch, K., and Remschmidt, H. (2010). Evidence for the involvement of genetic variation in the oxytocin receptor gene (OXTR) in the etiology of autistic disorders on highfunctioning level. Am. J. Med. Genet. B Neuropsychiatr. Genet. 153B, 629-639.
Wu, S., Jia, M., Ruan, Y., Liu, J., Guo, Y., Shuang, M., Gong, X., Zhang, Y., Yang, X., and Zhang, D. (2005). Positive association of the oxytocin receptor gene (OXTR) with autism in the Chinese Han population. Biol. Psychiatry 58, 74-77.

Zöllner, S., and Pritchard, J. (2007). Overcoming the winner's curse: estimating penetrance parameters from case-control data. Am. J. Hum. Genet. 80, 605-615.

Conflict of Interest Statement: The authors declare that the research was conducted in the absence of any commercial or financial relationships that could be construed as a potential conflict of interest.
Received: 11 October 2011; accepted: 16 January 2012; published online: $02 \mathrm{Feb}$ ruary 2012.

Citation: Krueger F, Parasuraman $R$ Iyengar $V$, Thornburg $M$, Weel J, Lin $M$, Clarke E, McCabe $K$ and Lipsky RH (2012) Oxytocin receptor genetic variation promotes human trust behavior. Front. Hum. Neurosci. 6:4. doi: 10.3389/fnhum.2012.00004 Copyright (c) 2012 Krueger, Parasuraman, Iyengar, Thornburg, Weel, Lin, Clarke, McCabe and Lipsky. This is an open-access article distributed under the terms of the Creative Commons Attribution Non Commercial License, which permits non-commercial use, distribution, and reproduction in other forums, provided the original authors and source are credited. 


\section{APPENDIX \\ DESCRIPTIONS OF PSYCHOLOGICAL CONTROL MEASURES Empathy}

The interpersonal reactivity index (IRI; Davis, 1983) contains four sub-scales, each tapping into a separate facet of empathy: the perspective taking scale assessing the reported tendency to spontaneously adopt the psychological point of view of others in everyday life (e.g., I sometimes try to understand my friends better by imagining how things look from their perspective), the empathic concern scale assessing the tendency to experience feelings of sympathy and compassion for unfortunate others (e.g., I often have tender, concerned feelings for people less fortunate than me), the personal distress scale assessing the tendency to experience distress and discomfort in response to extreme distress in others (e.g., Being in a tense emotional situation scares me), and the fantasy scale assessing the tendency to imaginatively transpose oneself into fictional situations (e.g., When I am reading an interesting story or novel, I imagine how I would feel if the events in the story were happening to me). Subjects were asked to read statements $(n=28)$ about their thoughts and feelings in a variety of situations and to decide how well each statement describes them on a five-point Likert scale $(1=$ strongly disagree to $5=$ strongly agree). A total score for empathy was derived by taking the mean across the four sub-scales.

\section{Theory of mind ability}

The reading the mind in the eyes test (RMET; Baron-Cohen et al., 2001) is a standardized multiple-choice test that assesses individual differences in the ability to infer the mental states of strangers. The RMET contains black-and-white photos $(n=36)$ of the eye region of different individuals. Each photo displays a particular cognitive or affective-state and is paired with four affective-state adjectives as response options (e.g., terrified, upset, arrogant, and annoyed). Subjects were asked to select the adjective that in their judgments best describes what the individual in the photo is feeling or thinking. A total score for theory of mind ability was derived by calculating the percentage of correct answers.

\section{Altruism}

The Rushton altruism scale (RAS; Rushton et al., 1981) is a selfreport scale that assesses individual tendency to altruistic behaviors. Subjects were asked to read statements $(n=20)$ describing altruistic behaviors (e.g., I have donated goods or clothes to a charity) and to indicate the frequency with which they carry out those behaviors on a five-point Likert scale ( $1=$ never to $5=$ very often $)$. A score for altruism was derived by taking the sum of ratings of all statements.

\section{Attachment style}

The Relationship Scale Questionnaire (RSQ; Griffin and Bartholomew, 1994) is a self-report scale that assesses four attachment styles: secure, dismissive, preoccupied, and fearful. Subjects were asked to read statements $(n=30$; e.g., I worry that I will be hurt if I allow myself to become too close to others) and to rate the extent to which they believe those statements best describes their feelings about close relationships on a five-point Likert scale $(1=$ not at all like me to $5=$ very much like me). Scores for each attachment style were derived by taking the mean of ratings for each attachment prototype.

\section{Personality style}

The NEO five-factor inventory (NEO-FFI; Costa and McCrae, 1992) is a psychological personality inventory of the Five-Factor Model: extraversion, agreeableness, conscientiousness, neuroticism, and openness to experience. Subjects were asked to read statements (e.g., I work hard to accomplish my goals) and to rate the extent to which those statements $(n=60)$ represents their opinion on a five-point Likert scale $(1=$ strongly disagree, $5=$ strongly agree). Scores for each personality style were derived by calculating the $T$-score (i.e., low average: $43-47$, average: $48-52$, high average: 53-57) for each of the five-factors. 
Table A1 | Investor's money transfers (mean \pm SD) and trustee' money back transfers (monetary units, MU) for the GG and AG/AA groups at each trust level.

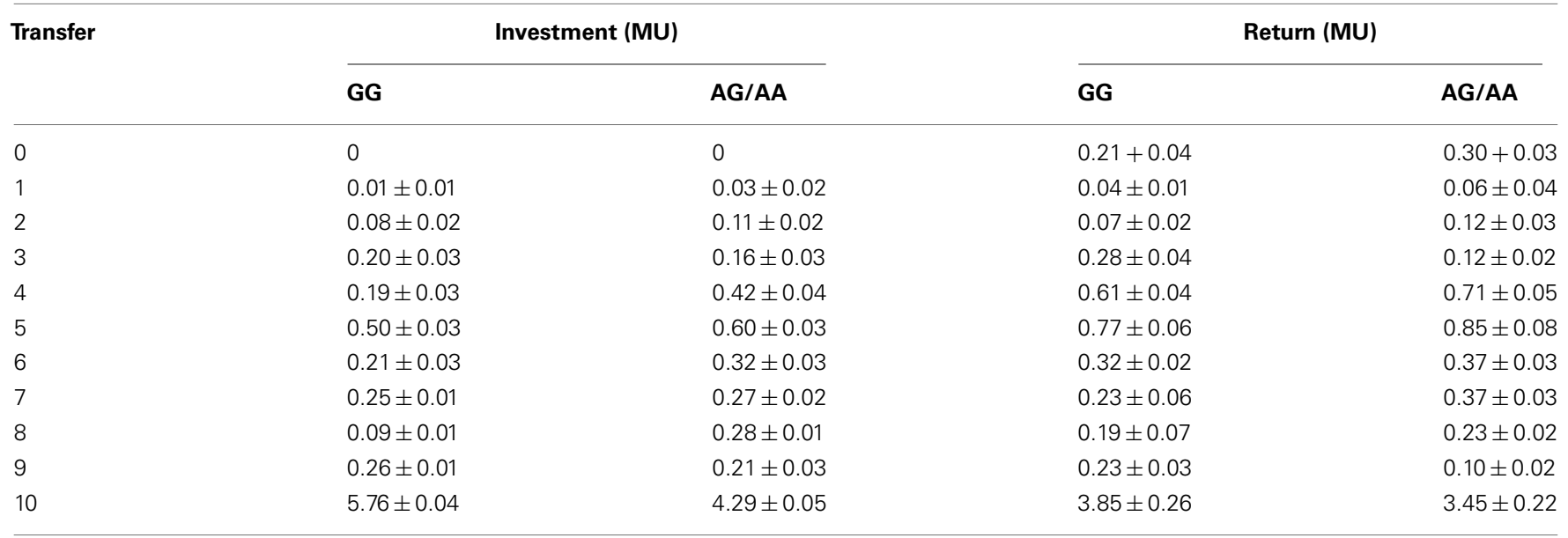
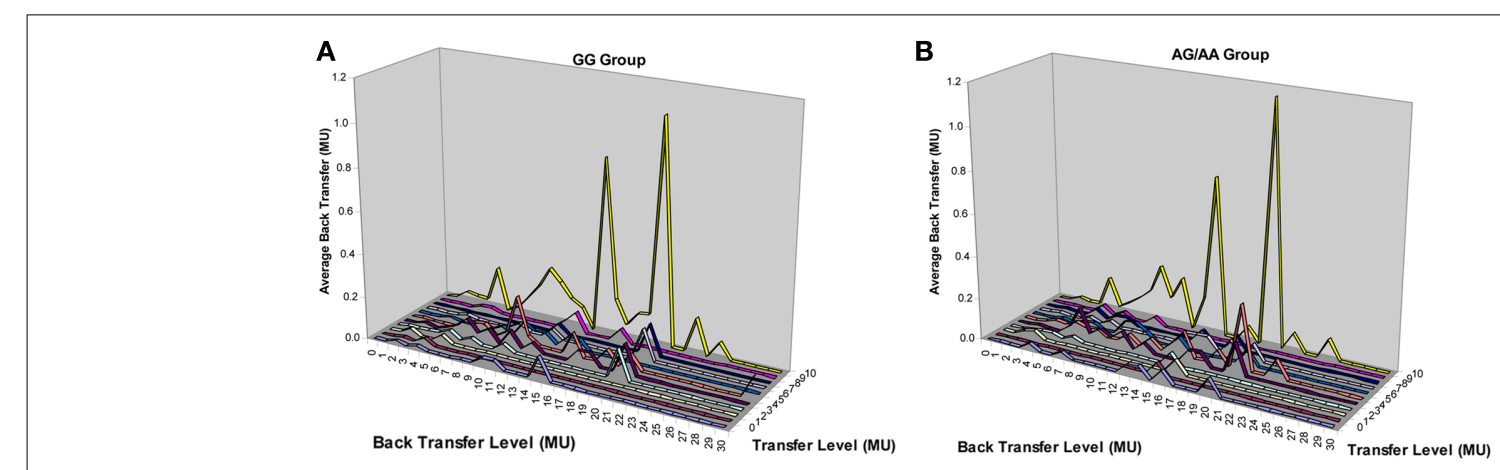

FIGURE A1 | Distribution of trustee's money back transfers in the trust experiment. (A) Average back transfers in the GG group at each transfer and back transfer level. (B) Average back transfers in the AG/AA group at each transfer and back transfer level. MU, monetary unit. 